\title{
Early response to neoadjuvant chemotherapy helps decrease recurrence rate of cervical cancer: a systematic review and meta- analysis
}

\author{
Qingxia Hou ${ }^{1 \#}$, Jian Shen ${ }^{2 \#}$, Su Zhou ${ }^{3}$, Jia Wei $^{3}$, Meng Wu ${ }^{3}$, Qian Chen ${ }^{3}$, Wei Yan ${ }^{3}$, Pei Zhang ${ }^{1}$, \\ Qingfen Yue ${ }^{1}$, Shixuan Wang ${ }^{3}$, Kecheng Huang ${ }^{3}$
}

${ }^{1}$ Department of Obstetrics and Gynecology, Luoyang Central Hospital Affiliated to Zhengzhou University, Luoyang, China; ${ }^{2}$ Department of Obstetrics and Gynecology, The Central Hospital of Wuhan, Tongji Medical College, Huazhong University of Science and Technology, Wuhan, China; ${ }^{3}$ Department of Obstetrics and Gynecology, Tongji Hospital, Tongji Medical College, Huazhong University of Science and Technology, Wuhan, China

Contributions: (I) Conception and design: Q Hou, K Huang, J Shen, Q Yue, S Wang; (II) Administrative support: Q Yue, S Wang; (III) Provision of study materials or patients: Q Hou, K Huang, J Shen; (IV) Collection and assembly of data: Q Hou, J Shen, S Zhou, J Wei, Q Chen, W Yan, M Wu, P Zhang; (V) Data analysis and interpretation: Q Hou, K Huang, J Shen; (VI) Manuscript writing: All authors; (VII) Final approval of manuscript: All authors.

\#These authors contributed equally to this work.

Correspondence to: Qingfen Yue. Department of Obstetrics and Gynecology, Luoyang Central Hospital Affiliated to Zhengzhou University, Luoyang 471009, China; Email: qingfenyue2006@126.com; Shixuan Wang. Department of Obstetrics and Gynecology, Tongji Hospital, Tongji Medical College, Huazhong University of Science and Technology, Wuhan 430030, China. Email: shixuanwang@tjh.tjmu.edu.cn; Kecheng Huang. Department of Obstetrics and Gynecology, Tongji Hospital, Tongji Medical College, Huazhong University of Science and Technology, Wuhan 430030, China. Email: kchuang@tjh.tjmu.edu.cn.

Background Neoadjuvant chemotherapy has been used for treatment of cervical cancer for a long time; however, the role of early non-response on prognosis is still confusing. This study was designed to assess its impact on disease-free survival (DFS).

Methods: Databases "PubMed", "Embase" and the "Cochrane Library" were searched out through May 2020, and both random effects model and fixed effect model were employed to calculate the main pooled results. $\mathrm{I}^{2}$ and Cochrane $\mathrm{Q}$ test were used to test the heterogeneity among the studies. Funnel plot with Begg's and Egger's tests was used to assess the publication bias that may exist in the study. Sensitivity analysis was performed to detect the origin of the heterogeneity.

Results: A total of 1,349 articles were found at first; then, after several rounds of exclusion, we identified 8 articles with 9 studies which were accordant with the standards of the inclusion. A combined analysis was performed among the 1,462 responders and 490 non-responders. For 1-year DFS, sub-analysis showed hazard ratio (HR) was 0.25 (95\% CI: 0.14-0.43) using RECIST criteria; and HR was 0.52 (95\% CI: 0.360.75 ) using WHO criteria; Egger's test showed that $\mathrm{P}=0.35$ for RECIST criteria and $\mathrm{P}=0.57$ for WHO criteria; Begg's test showed $\mathrm{P}=0.34$ for RECIST criteria and $\mathrm{P}=0.60$ for WHO criteria. For 3-year DFS, HR was 0.26 (95\% CI: 0.16-0.43) using RECIST criteria and was 0.47 (95\% CI: 0.30-0.73) using WHO criteria. For 5-year DFS, HR was 0.26 (95\% CI: 0.16-0.42) using RECIST criteria and was 0.49 (95\% CI: 0.33-0.71) using WHO criteria.

Discussion: Early non-response to neoadjuvant chemotherapy was significantly associated with higher recurrence of cervical cancer. Prospective randomized studies are warranted to validate this finding.

Keywords: Early response; meta-analysis; neoadjuvant chemotherapy (NACT); recurrence; uterine cervical neoplasms

Submitted Oct 11, 2020. Accepted for publication Apr 15, 2021.

doi: 10.21037/apm-20-2004

View this article at: http://dx.doi.org/10.21037/apm-20-2004 


\section{Introduction}

Cervical cancer has been one of the most common malignant disease in undeveloped areas (1). It has been estimated that there are 98,900 incidences and 30,500 death in 2015 in China, which is also the top malignant tumor in the field of obstetrics and gynecology (2). Nowadays, clinicians have resorted to neoadjuvant chemotherapy (NACT) plus surgery for the treatment of cervical cancer. And there are several reasons: firstly, NACT can reduce the tumor size, diminish distant metastasis, and facilitate the surgery; secondly, instead of radiotherapy, NACT plus surgery provides the opportunity to have the vaginal function and ovarian function preserved, and patients consequently enjoy better life quality (3). Thirdly, for young women who are eager to have their reproductive function preserved, NACT provides the chance of genital preservation (4-6). Fourth, for women who are pregnant, NACT provides the chance to prolong the duration of pregnancy (7). Thus, NACT has widely been used across the world (8).

Recently, quite a number of studies have investigated the prognostic role of NACT's short-term response on longterm survival. However, the results are always disputing (9). Therefore, the present study is designed aiming to give a pooled conclusion by using the published data from the previous studies.

\section{Methods}

\section{Literature searching}

This systematic review was carried out according to the disciplines of the PRISMA guidelines as well as MOOSE guidelines $(10,11)$ (available at http://dx.doi. org/10.21037/apm-20-2004). Literature was searches out until May 2020 by two doctors (J Shen and Q Hou) in our team independently. The English databases, "PubMed", "Embase" and the "Cochrane Library" were searched at the beginning of the present research. The team performed the searching by using the items "preoperative chemotherapy", "NACT", "neoadjuvant chemotherapy", plus "response" or "responder" or "responding" or "responsiveness" or "clinical response" or "remission", plus "cervical cancer" or "cervical carcinoma" or "cervical neoplasia". To avoid the data missing, the reference articles in the retrieved articles were also reviewed.

\section{Eligibility criteria and data screening}

Three rounds of identification were adopted to collect the necessary studies in the present research. Articles with cervical cancer and therapy were firstly searched out. During the first screening, articles concerning about cervical cancer and chemotherapy were included by reading the titles and abstracts of the articles; otherwise, they would be excluded. During the second screening, articles focusing on cervical cancer and NACT were excluded by reading the result section of the papers as well as the supplementary materials. Meanwhile, the selected articles must fulfill all of our criteria: the articles must be written in English; the articles must be original research articles; all cases in the articles were definitely diagnosed with cervical carcinoma; the included articles must be published in the journals following peer-review disciplines. During the third screening, articles with disease-free survival (DFS) data were included in the final analysis. Newcastle-Ottawa Scale (NOS) was adopted to evaluate the quality of the included studies (12).

\section{Data extraction}

Hazard ratio (HR) and corresponding 95\% CI were extracted if they were provided in the articles. If HR was not provided in the articles, software was used to read the survival curves according to the methods reported in the previous studies (13).

\section{Statistical analysis}

According to the previous studies, responder was defined as clinical response $(\mathrm{CR})+$ partial response $(\mathrm{PR})$; at the same time, non-responder was defined as stable disease (SD) + progressive disease (PD). Both RECIST criteria and WHO criteria were adopted to evaluate the CR by previous studies, so in this study we also investigated both criteria. In WHO criteria, tumor response was judged according to bidimensional measurements; in RECIST criteria, tumor response was judged by one dimension measurement. There were slight differences between the two response criteria (14). Both criteria were widely accepted as standard methods in assessing the CR among the field of solid tumor research as well as among cervical cancer.

As described above, the relationship between the CR and 
long-term DFS was measured by HR with $95 \%$ confidential interval (CI). For the studies that HRs and 95\% CI could not directly be read from the articles, Engauge Digitizer software was used to read the survival curves and then to give the definite HRs (13). HRs were found from the main text, the tables or the supplementary materials in 5 studies, while survival curves were read from the other 4 studies. The RECIST criteria were found to be used in 6 studies and the WHO criteria were found in 3 studies (15-22). Forest plot was used to illustrate the pooled result for the HR and the corresponding 95\% CI. During the process, $\mathrm{I}^{2}$ statistic was adopted in our study to evaluate the heterogeneity that may exist in the studies; if $\mathrm{I}^{2}$ got the value larger than $50 \%$, the statistically significant heterogeneity was observed and then the random effect model was used in the study instead of the fixed effect model (23). Meanwhile, Cochrane $Q$ test was also used to assess the heterogeneity; and the fixed effect model should not be adopted when statistical heterogeneity was observed; in this condition, the researchers were obliged to use the random effect model. Funnel plot was employed to display and visually spot the publication bias that may exist during pooling across the studies. As funnel plot was unable to give a definite conclusion, non-parametric test (Begg's test) and parametric test (Egger's test) were also used in the study to detect the publication bias (24-26). Sensitivity analysis was also employed to test the robustness of the pooling result; and also to detect the origin of the heterogeneity. The R statistical software was used to perform the statistical analysis. Unless it was particularly noted in the context, $\mathrm{P}<0.05$ (two-sided) was regarded as statistically significant.

\section{Results}

\section{Literature search}

Articles were search out through May 2020 using the key items described in the section of methods. At the beginning, 1,349 articles were found by reading the titles and the abstracts. Then, by reading the context of the manuscript, articles concerning about cervical cancer, NACT and short-term response were identified; on the contrast, if these items were not studied both at the main text and the supplementary materials in the context, articles will be excluded; thus, 16 articles were retained. At last, articles will also be excluded unless they concern about the long-term DFS; thus, 8 articles including 9 studies were identified in the present research, and were finally used for pooling.
Details were shown in Figure 1.

\section{The relationship between short-term response and DFS}

\section{Characteristics of the included studies}

Table 1 listed the characteristics of the included studies. WHO criteria were adopted in 3 studies, while RECIST criteria were adopted in 6 studies. Cox proportional hazard regression models were observed in 5 studies, and KaplanMeier (K-M) curves were observed in 4 studies. The total 9 studies included 1,952 patients with 1,462 responders and 490 non-responders. There were 3 prospective studies and 6 retrospectives studies. The combined results based on RECIST criteria and WHO criteria were separately calculated.

\section{HR for 1-year DFS}

For all the studies adopting RECIST criteria, which included both prospective and retrospective studies, the fixed effect model gave a combined result of HR equaling to 0.25 (95\% CI: 0.14-0.43); Cochrane Q test didn't reveal that obvious heterogeneity existed across the studies $(\mathrm{P}=0.92)$. Similar result was also identified by $\mathrm{I}^{2}$ test as it was reported with the value of $0.0 \%$. Random effect analysis was also employed to calculate the weight of the corresponding studies ( $\mathrm{HR}=0.25$ ). Both fixed effect model and random effects model were listed in the same illustration (Figure 2). Funnel plot was made to visually screen the publication bias that may exist among the studies (Figure 3). Trim-andfill method was adopted from 1-year DFS to 5-year DFS (Figure 4) and it showed $\mathrm{P}<0.001$ with 1 added study for the 1 -year DFS (Figure 4A). Results showed that $\mathrm{P}$ equaled to 0.3499 for Egger's test (Figure S1) and P equaled to 0.3476 for Begg's test (Figure S2). Subgroup analysis for WHO criteria response and DFS was also performed. Trim-andfill plot was shown as Figure $4 B$; Egger's test was shown as Figure S1B; Begg's test was shown as Figure 2B. Sensitivity analysis was also made to test the robustness of the study, and the result showed that all the lower limit of the confidence intervals was not less than 0.52 (Figures S3,S4).

\section{HR for 3-year DFS}

Subgroup analysis was performed among the studies using both RECIST criteria and WHO criteria. For studies using RECIST criteria, both fixed effect model and random effect model were employed to calculate the total HR. But no difference was observed between the two models, as no obvious heterogeneity was detected across the studies. 


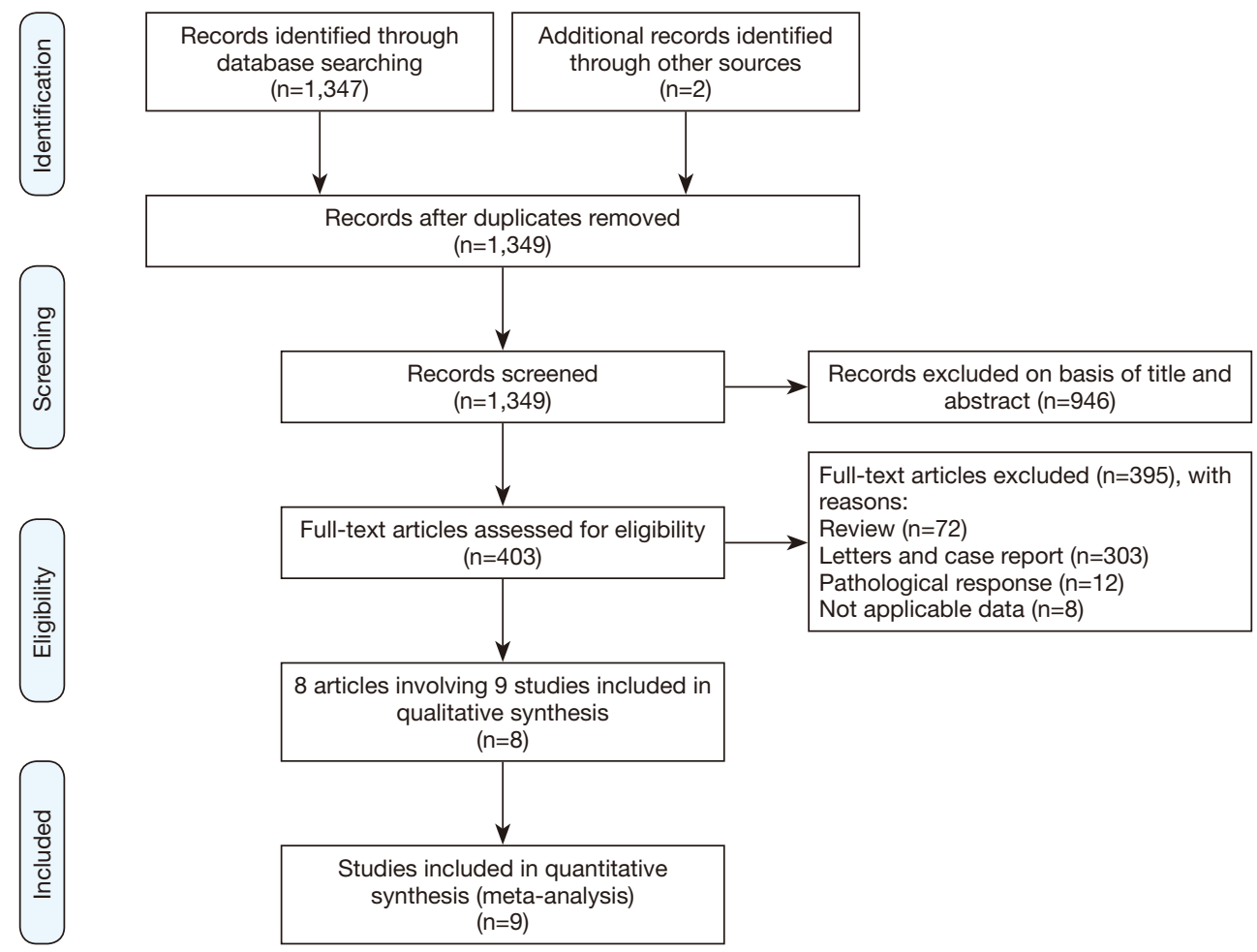

Figure 1 PRISMA 2009 flow diagram. PRISMA 2009 flow diagram with exclusion criteria. PRISMA, preferred reporting items for systematic reviews and meta-analyses.

Funnel plot was made to publication bias was visually screened by funnel plot (Figure 3C,D). Trim-and-fill plot was also used (Figure 4C,D). Cochrane $Q$ test revealed $\mathrm{P}=0.51$, and $\mathrm{I}^{2}$ test showed the value equaled to 0 . For studies adopting $\mathrm{WHO}$ criteria, the pooled HR was 0.49 (95\% CI: 0.35-0.69) when fixed effect model was employed; and the pooled HR was 0.47 (95\% CI: $0.30-0.73$ ) when random effect model was employed (Figure 5); parametric test like Egger's test (Figure S1C,D) and non-parametric test like Begg's test (Figure S2C,D) were also employed. And the result for sensitivity analysis was shown at Figures S5,S6.

\section{HR for 5-year DFS}

Both RECIST and WHO criteria were included in the analysis. The pooled result after the combination of the studies is shown at the bottom of the forest plot. When they were pooled together, funnel plot was adopted to adjust for funnel plot asymmetry (Figure $3 E, F$ ). Plots using Trimand-fill method was also used (Figure 4E,F). For RECIST criteria, the analysis showed a combined result with HR equaling to 0.26 (95\% CI: 0.16-0.42). A Cochrane $Q$ test produced a $\mathrm{P}$ value of 0.64 , and $\mathrm{I}^{2}$ test showed the value equaled to $0 \%$. For WHO criteria, HR was 0.50 (95\% CI: 0.36-0.69) when fixed effect model was used, while HR was 0.49 (95\% CI: $0.33-0.71)$ when random effect model was used (Figure 6). One of them was from Europe and the rest two studies were from Asia. Parametric test using Egger's method (Figure S1E,F) and non-parametric test like Begg's method (Figure S2E,F) were also employed. And the result for sensitivity analysis was shown at Figures S7,S8.

\section{Discussion}

In the present research, we combined all the published studies that focused on the short-term response and DFS. The WHO criteria and the RECIST criteria were separately investigated to give their own pooled results. Both fixed effect model and random effect model were used; these models also helped us to detect the heterogeneity across the studies. With the help of the database (27), the duplicate data was accurately checked out and then excluded, which led to a more solid result.

NACT has emerged as adjuvant therapy since two 
Table 1 Characteristics of the included studies

\begin{tabular}{|c|c|c|c|c|c|c|c|c|c|c|c|c|c|c|c|}
\hline Study & Publishing time & Location & Study period & Population & Data type & $\begin{array}{l}\text { No. of cases (non- } \\
\text { responders) }\end{array}$ & $\begin{array}{l}\text { No. of all } \\
\text { patients }\end{array}$ & Age at baseline, $y$ & Chemotherapy courses & FIGO stage & $\begin{array}{l}\text { Response evaluation } \\
\text { guidelines }\end{array}$ & $\begin{array}{l}\text { Using K-M for } \\
\text { data extraction } \\
\text { or not }\end{array}$ & $\begin{array}{l}\text { Effect size from } \\
\text { Cox model or } \\
\text { not }\end{array}$ & Adjustment & Follow-up period \\
\hline Xie (22) & 2016 & China & 2003-2008 & Chinese & Retrospective & 18 & 52 & $\begin{array}{l}\text { Median [range]: } 43 \\
{[27-63]}\end{array}$ & $2-3$ & IB2-IIB & RECIST criteria & No & Yes & $\begin{array}{l}\text { Tumor size, the expression of } \\
\text { ALDH1 }\end{array}$ & 3-123 months \\
\hline Yang (18) & 2015 & China & 2007-2012 & Chinese & Retrospective & 33 & 115 & $\begin{array}{l}\text { Median [range]: } 45 \\
\text { [23-68) }\end{array}$ & Unknown & IB2-IIB & RECIST criteria & Yes & No & None & $6-75$ months \\
\hline Park (19) & 2011 & Korea & 1997-2007 & Korean & Prospective & 15 & 28 & $\begin{array}{l}\text { Median [range]: } 50 \\
{[30-78]}\end{array}$ & $\begin{array}{l}\text { Most of patients } \\
\text { received three courses } \\
\text { of NACT }\end{array}$ & IIB & RECIST criteria & No & Yes & Node, the expression of ERCC1 & 6-139 months \\
\hline Shoji (21) & 2013 & Japan & 2002-2011 & Japanese & Retrospective & 5 & 23 & $\begin{array}{c}\text { Median [range]: } 50 \\
\text { [32-63] }\end{array}$ & $1-3$ & IB2-IIB & RECIST criteria & No & Yes & None & 9-90 months \\
\hline Li [1] (15) & 2016 & China & 1999-2008 & Chinese & Retrospective & 189 & 826 & $\begin{array}{c}\text { Median [range]: } 44 \\
\text { [39-50] }\end{array}$ & Mostly $1-2$ & IB2-IIB & WHO criteria & No & Yes & $\begin{array}{l}\text { Age, stage, tumor size, grade, cell } \\
\text { type, LVSI, parametrial infiltration, } \\
\text { vaginal surgical margin, Iymph node } \\
\text { metastasis }\end{array}$ & 0-115 months \\
\hline Li [2] (15) & 2016 & China & 2003-2013 & Chinese & Prospective & 115 & 485 & $\begin{array}{c}\text { Median [range]: } 45 \\
\text { [40-49] }\end{array}$ & Mostly 1-2 & IB2-IIB & WHO criteria & No & Yes & $\begin{array}{l}\text { Age, stage, tumor size, grade, cell } \\
\text { type, LVSI, parametrial infiltration, } \\
\text { vaginal surgical margin, Iymph node } \\
\text { metastasis }\end{array}$ & $0-100$ months \\
\hline
\end{tabular}

SD, standard error: NACT, neoadjuvant chemotherapy; K-M, Kaplan-Meier; RECIST, Response Evaluation Criteria in Solid Tumors: FIGO, International Federation of Gynecology and Obstetrics: LVSI, lymphovascular space invasion. 


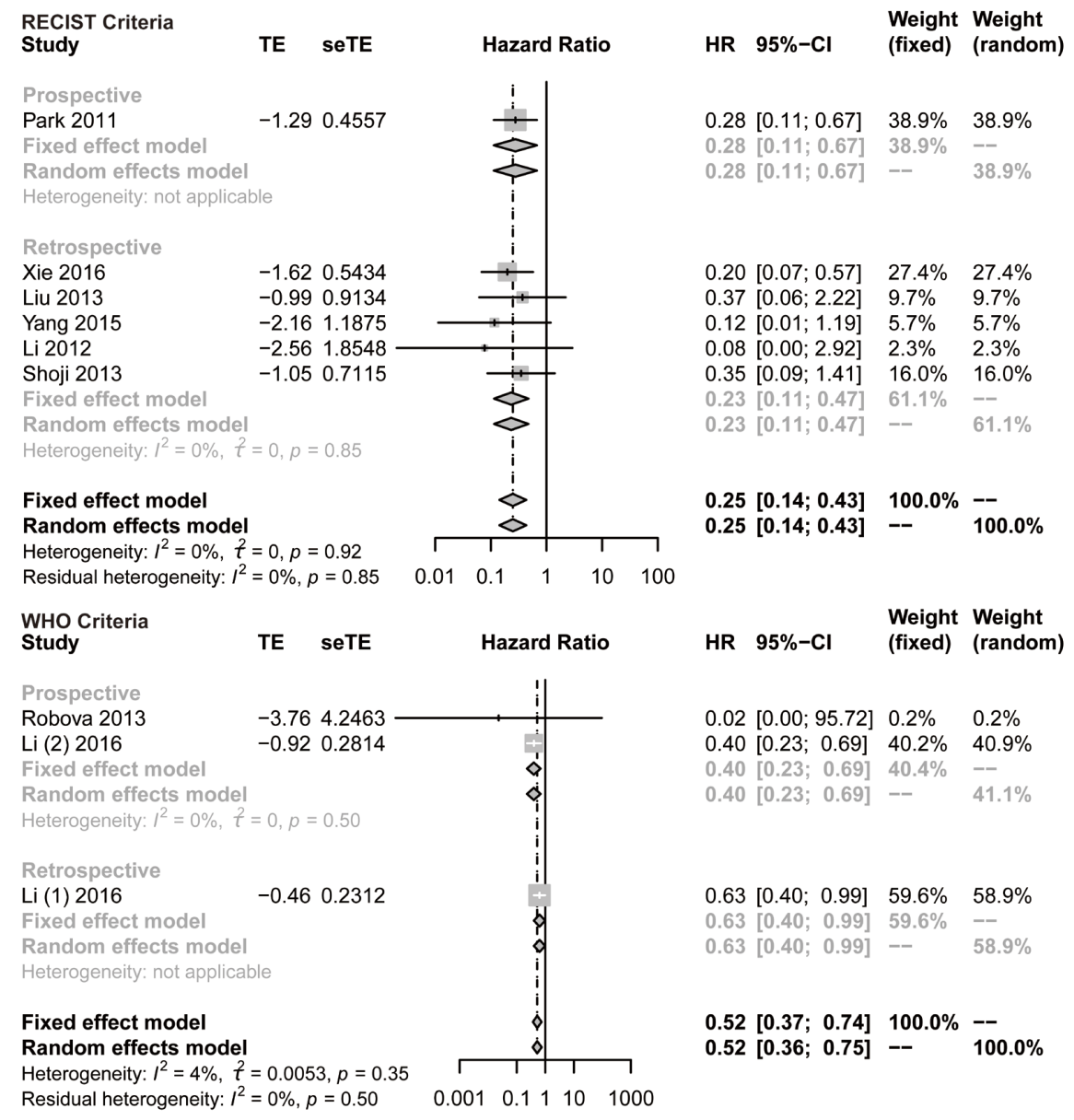

Figure 2 The pooling HR for 1-year DFS. The summary estimates were obtained by using both fixed-effect and random-effects model. The data markers indicate the HRs comparing non-responder with responder. The size of the data markers indicates the weight of the study, which is the inverse variance of the effect estimate. The diamond data markers indicate the pooled HR. HR, hazard ratio; CI, confidence interval; DFS, disease-free survival.

decades of years ago; and with surgery, it has been employed as an alternative therapy for traditional radiotherapy (28). Recently, its therapeutic role has been investigated by more and more scholars across the world. Studies showed its potential effect on replacing traditional methods for young patients undergoing cervical cancer, as patients got the chance to have their fertility preserved (4). For patients in pregnancy, NACT has brought the new hope to give birth to a baby for it can disrupt the tumor's growth, even make the tumor diminish, and thus prolong pregnancy period (7). For women who don't want to suffer from radiation, NACT plus surgery provide the chance to have their vaginal function preserved and thus they can enjoy a high level of quality for their life after they are cured $(4,5)$. According to the previous research, there is one thing that should be particularly paid attention to: DFS is quite different from overall survival (OS), and NACT can help reduce tumor size and metastasis, but may not be able to have all patients' OS rate increased; the prognostic effect of early response on OS still needed to be exactly looked through after all the literature was carefully extracted.

We have made similar findings with other researchers' studies. MacLeod and colleagues conducted a single institutional study and found that responsiveness was a predictor of survival in univariate analysis using Cox proportional hazard regression model, with most of the patients achieving an optimal response (complete response plus PR) (29). Cai and colleagues conducted a prospective 
A

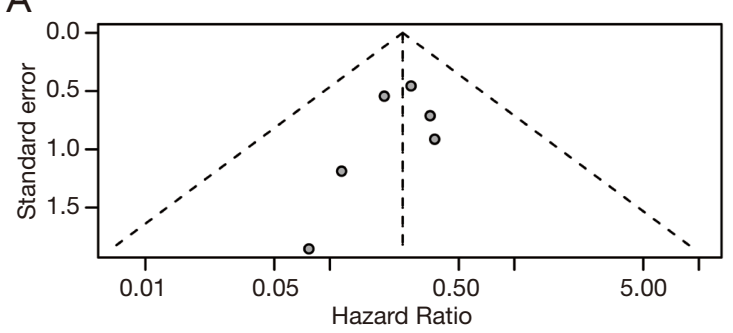

C

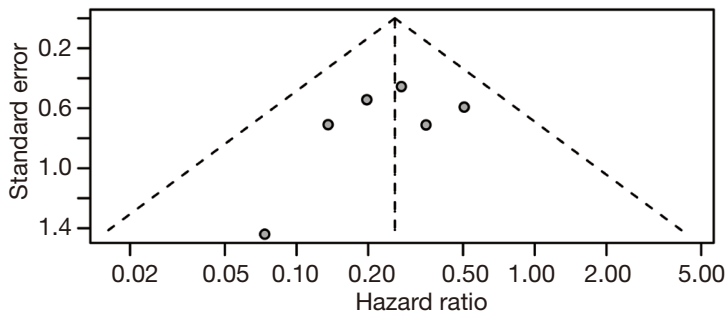

$\mathrm{E}$

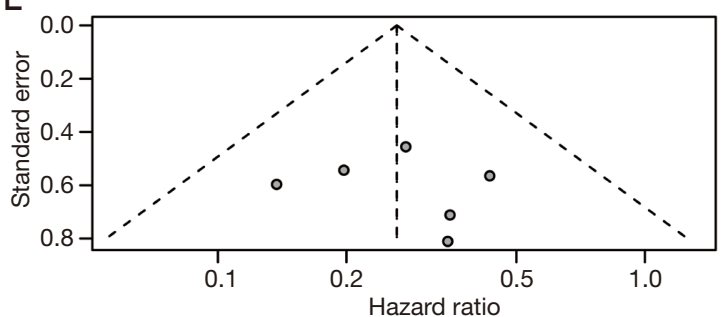

B

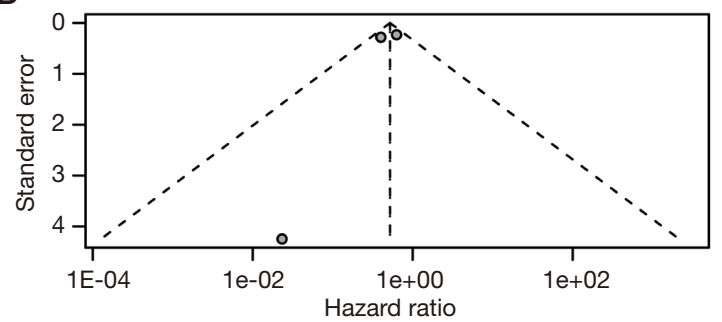

D

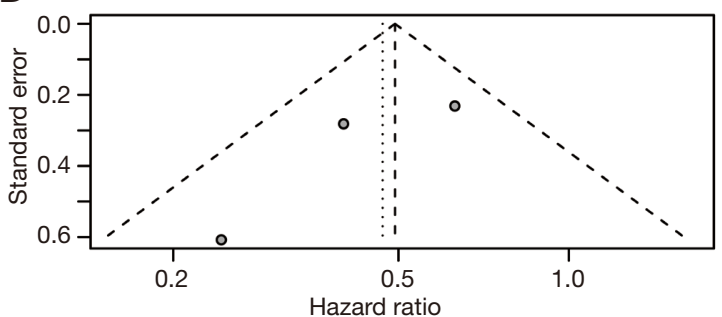

$\mathrm{F}$

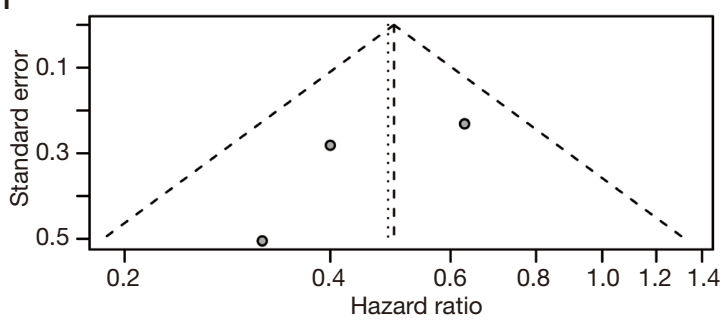

Figure 3 Funnel plot for detection of publication bias of HR for DFS. The pseudo 95\% CI is computed as part of the analysis that produces the funnel plot, and corresponding to the expected $95 \%$ CI for a given standard error. (A,B) The funnel plot of 1-year DFS for studies adopting RECIST criteria and WHO criteria, respectively; (C,D) the funnel plot of 3-year DFS for studies adopting RECIST criteria and WHO criteria, respectively; (E,F) represents the funnel plot of 5-year DFS for studies adopting RECIST criteria and adopting WHO criteria, respectively. HR, hazard ratio; DFS, disease-free survival; CI, confidence interval.

randomized study with NACT, and early clinical nonresponsiveness rate was $15.4 \%$; meanwhile, they found that the survival rate of early non-responders was significantly lower than partial responders plus complete responders with statistically significance $(\mathrm{P}=0.0049)$; at the same time, they found that vascular space involvement rate was lower in NACT group than primary surgery group (30). Mori and colleagues conducted a study for NACT consisted of paclitaxel and carboplatin, and they observed that the patients who were non-responsive to NACT had the lowest survival rate of $50.0 \%$, whereas the survival rate of complete response was $100 \%$ and the survival rate of PR was $87.3 \%$ (31). Selvaggi and colleagues found that in univariate Cox regression analysis responsiveness to NACT was a significant prognostic factor of survival $(\mathrm{P}<0.0001)$; meanwhile, the K-M survival curves showed that the non-responders got lower survival rate compared with complete responders and partial responders (32). Martinelli and colleagues made a study on locally advanced cervical cancer (LACC) patients who underwent NACT, and they found that early responsiveness was an predictor of survival $(\mathrm{P}=0.001)$ and non-response was significantly associated with worse survival rate; at the same time, the result was still statistically significant with $\mathrm{P}$ value equaling to 0.001 even adjusted with the factors including pathological response, vaginal involvement, and lymph nodes metastasis; meanwhile, they found that the early nonresponsive patients got more chance to have parametrial involvement (33). Previous study conducted by other countries' team also showed that patients with LACC who received NACT treatment prior to the radical surgery were less likely to need postoperative radiation therapy.

The present finding validated the previous studies $(15,16)$. Quite a lot of studies made similar finding in the past two 

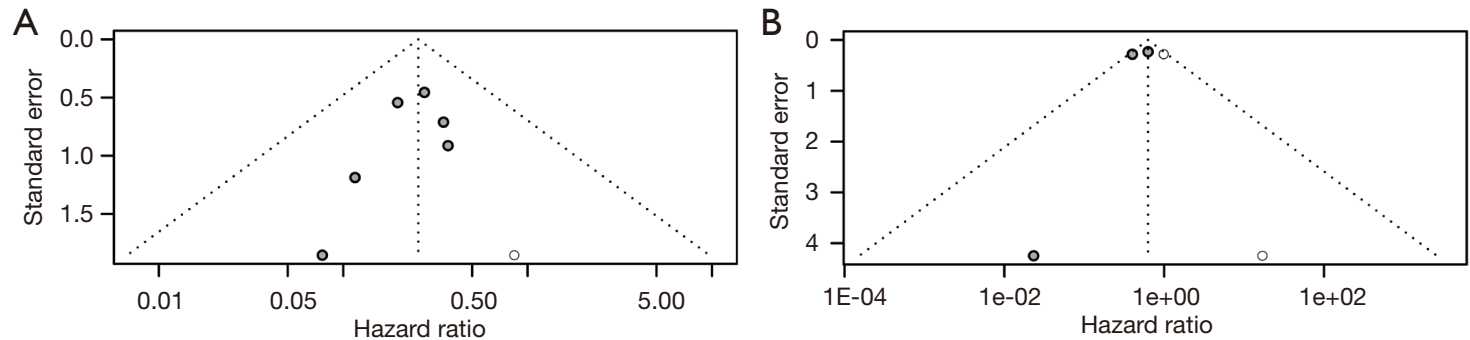

C
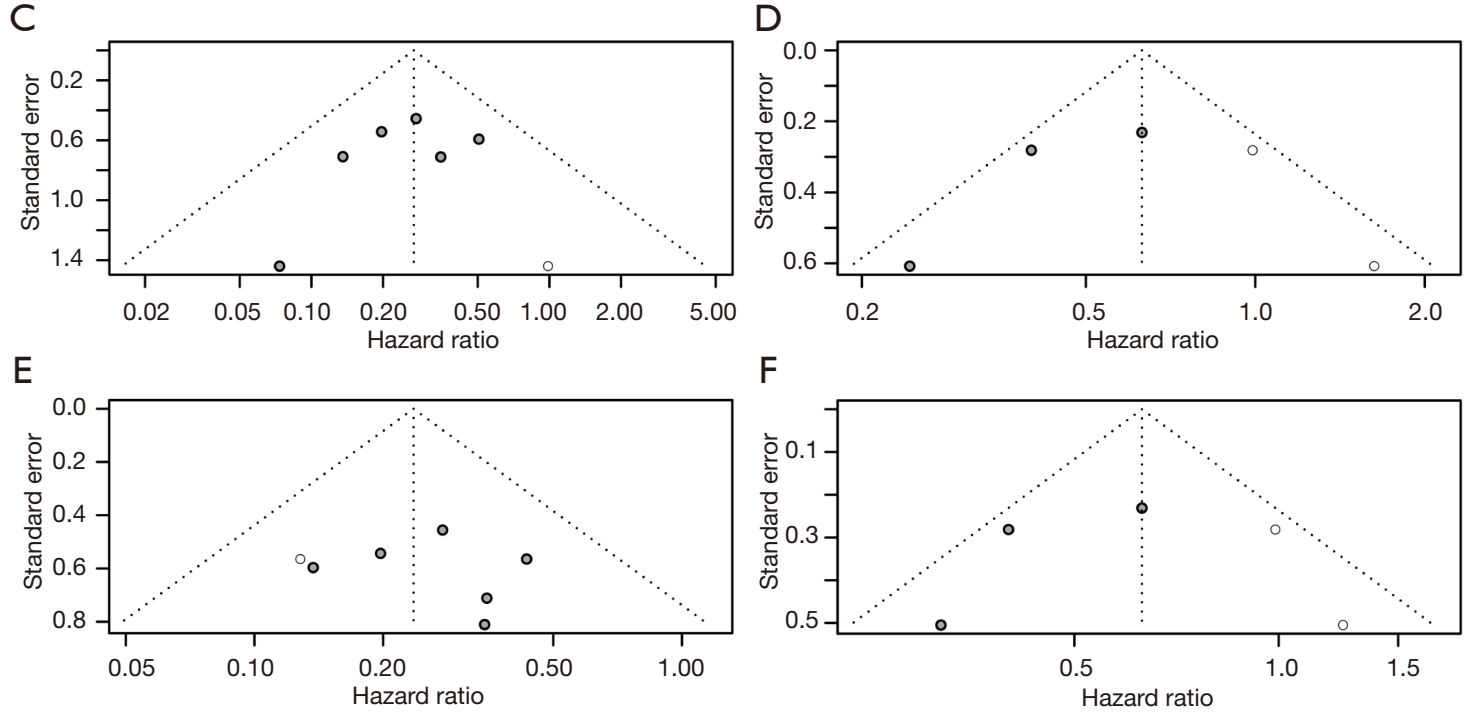

Figure 4 Trim-and-fill plot for detection of publication bias of HR. HR, hazard ratio. The pseudo 95\% CI is computed as part of the analysis that produces the funnel plot, and corresponding to the expected $95 \%$ CI for a given standard error; trim-and-fill plot was a nonparametric method for estimating the number of missing studies that might exist in a meta-analysis and the effect that these studies might have had on its outcome. (A,B) The 1-year DFS adopting RECIST criteria and WHO criteria, respectively; (C,D) the 3-year DFS adopting RECIST criteria and WHO criteria, respectively; (E,F) the 5-year DFS adopting RECIST criteria and WHO criteria, respectively. HR, hazard ratio; DFS, disease-free survival; CI, confidence interval.

decades $(18-20,22,34,35)$. Our finding is partly different from these findings made by other scholars in some of the previous studies. Liu and colleagues made a research from the year 2002 to 2011; however, they found that the survival rate between responders and non-responders showed no statistically difference. Shoji and colleagues also made a study on a group of 23 patients, and their founding was not consistent with the nowadays finding either. As a result, our team made a thorough look for all the studies on this issue, and we found several reasons for the inconsistency: first, the number of patients taking part in the study, as only enough sample size can give a solid conclusion with enough power; second, the follow-up years, was also important in giving a more accurate conclusion, especially when not enough patients were enrolled in the study. And in the present study, we used the proper method and joined all the studies together to give a final conclusion.

We also compared the 5-year OS between the responders and non-responders, and found that responders showed higher survival rates compared with non-responders (data not shown). For studies adopting RECIST criteria, the fixed effect model and random effect model showed the same result. As for WHO criteria, the fixed effect model and random effect model showed different results. When random effect model was used, and it revealed a pooled result of HR equaling to the previous finding. Significant heterogeneity was observed among the studies, as Cochrane $Q$ test showed that a random effect model should be employed. To screen the publication bias, funnel plot was made as well as Egger's test and Begg's test. Sensitivity analysis was also performed to test the robustness of the pooled result, and to find the origin of 


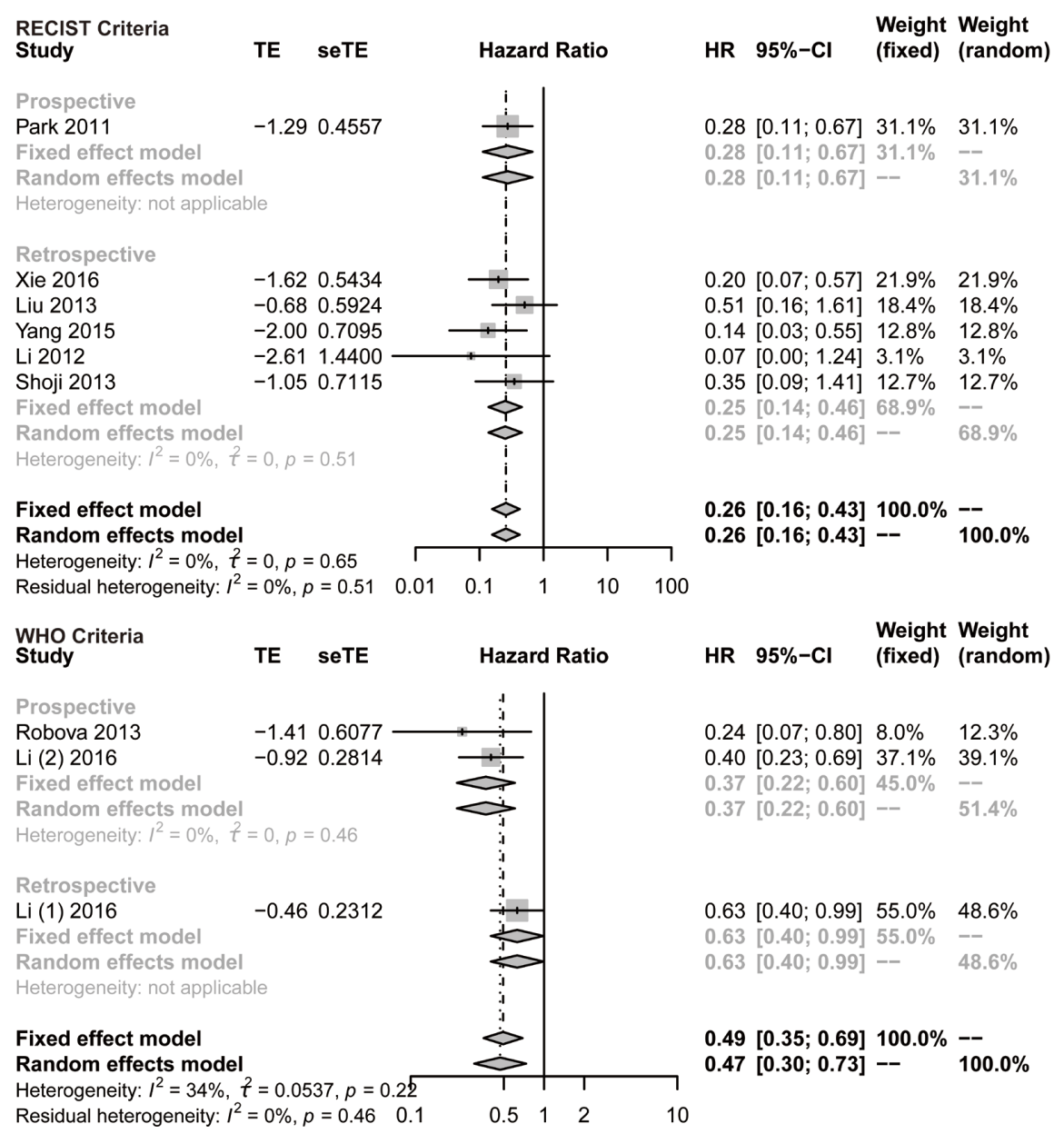

Figure 5 The pooling HR for 3-year DFS. The summary estimates were obtained by using both fixed-effect and random-effects model. The data markers indicate the HRs comparing non-responder with responder. The size of the data markers indicates the weight of the study, which is the inverse variance of the effect estimate. The diamond data markers indicate the pooled HR. HR, hazard ratio; CI, confidence interval; DFS, disease-free survival.

the heterogeneity.

And there are some limitations in our study. First, a novel predictor for long-term survival, optimal pathological response (OPR) was not investigated in our study (36,37); second, genetic or epigenetic studies based on population may be able to provide a clear explanation why some people are more susceptible to certain type of tumors while some people response to chemotherapy drugs better than the others, and which is not discussed in the present research (38). As a result, our team made a decision on the research looking through the predictive value of the novel makers including OPR, and it will be discussed in the near future in our following study. At the same time, we will do our best to contact the authors in our field and ask them for cooperating on the research for early response's prognosis on long-term survival.

\section{Conclusions}

In conclusion, we made a pooled analysis for identifying the predictive effect of early response on DFS. Both WHO criteria and RECIST criteria were investigated in the present study. And the statistical result showed that early response was actually a predictor of long-term survival. Responders evaluated by both WHO criteria and RECIST criteria were significantly associated with DFS. This finding may provide some clue in predicting the prognosis and in identifying the group of high-risk patients with cervical cancer. 


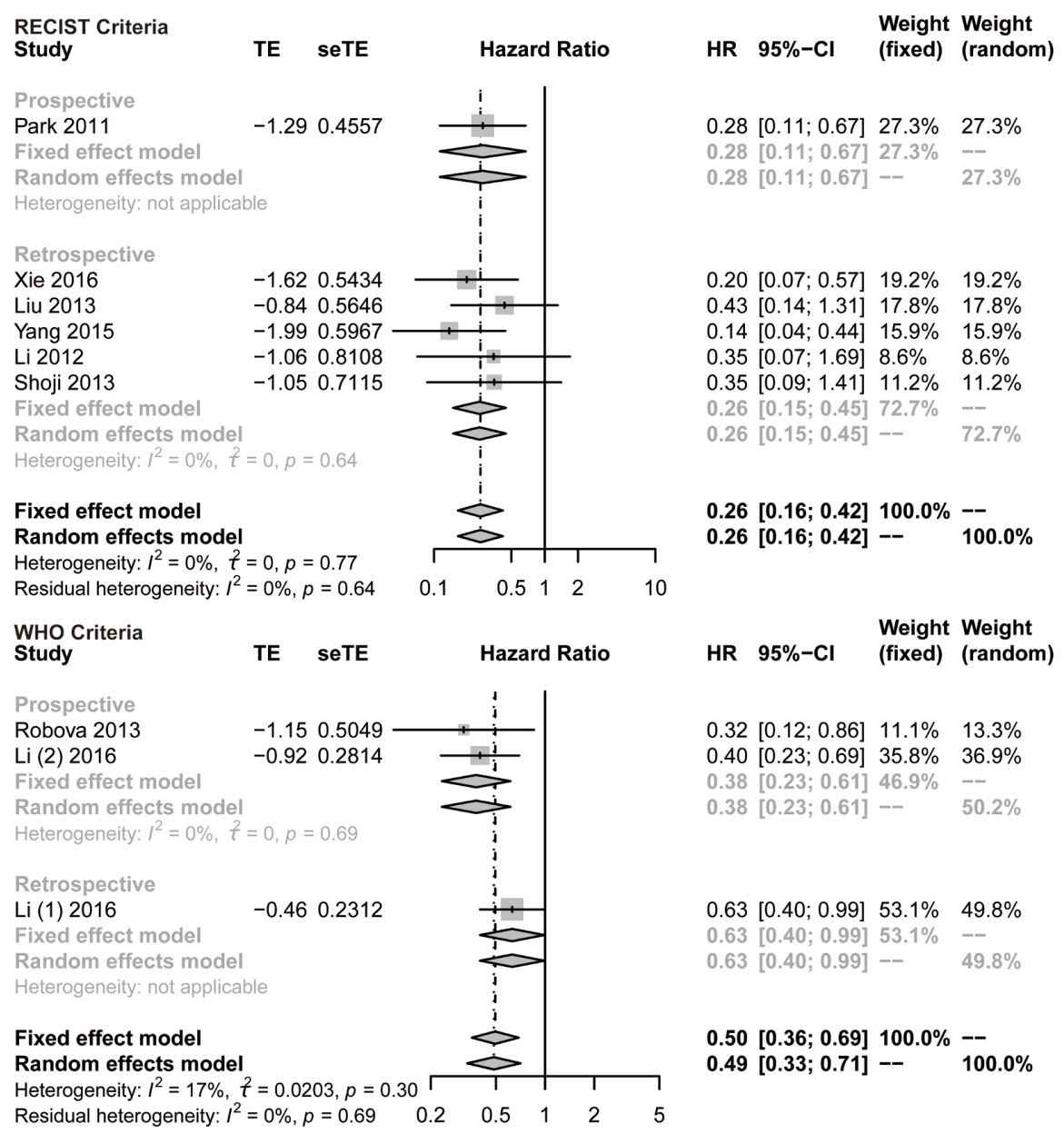

Figure 6 The pooling HR for 5-year DFS. The summary estimates were obtained by using both fixed-effect and random-effects model. The data markers indicate the HRs comparing non-responder with responder. The size of the data markers indicates the weight of the study, which is the inverse variance of the effect estimate. The diamond data markers indicate the pooled HR. HR, hazard ratio; CI, confidence interval; DFS, disease-free survival.

\section{Acknowledgments}

We thank the statisticians for their help with statistical analysis.

Funding: This work was supported by the grants from the National Key R\&D Program of China (2016YFC1302902) and Key program of Natural Science Foundation of Hubei Province (2016CFA064).

\section{Footnote}

Reporting Checklist: The authors have completed the PRISMA and MOOSE reporting checklists. Available at http://dx.doi.org/10.21037/apm-20-2004
Conflicts of Interest: All authors have completed the ICMJE uniform disclosure form (available at http://dx.doi. org/10.21037/apm-20-2004). The authors have no conflicts of interest to declare.

Ethical Statement: The authors are accountable for all aspects of the work in ensuring that questions related to the accuracy or integrity of any part of the work are appropriately investigated and resolved.

Open Access Statement: This is an Open Access article distributed in accordance with the Creative Commons Attribution-NonCommercial-NoDerivs 4.0 International License (CC BY-NC-ND 4.0), which permits the non- 
commercial replication and distribution of the article with the strict proviso that no changes or edits are made and the original work is properly cited (including links to both the formal publication through the relevant DOI and the license). See: https://creativecommons.org/licenses/by-nc-nd/4.0/.

\section{References}

1. Bray F, Ferlay J, Soerjomataram I, et al. Global cancer statistics 2018: GLOBOCAN estimates of incidence and mortality worldwide for 36 cancers in 185 countries. CA Cancer J Clin 2018;68:394-424.

2. Chen $W$, Zheng R, Baade PD, et al. Cancer statistics in China, 2015. CA Cancer J Clin 2016;66:115-32.

3. Sardi JE, Boixadera MA, Sardi JJ. Neoadjuvant chemotherapy in cervical cancer: a new trend. Curr Opin Obstet Gynecol 2005;17:43-7.

4. Rob L, Skapa P, Robova H. Fertility-sparing surgery in patients with cervical cancer. Lancet Oncol 2011;12:192-200.

5. Bentivegna E, Gouy S, Maulard A, et al. Oncological outcomes after fertility-sparing surgery for cervical cancer: a systematic review. Lancet Oncol 2016;17:e240-53.

6. Dolmans MM, Manavella DD. Recent advances in fertility preservation. J Obstet Gynaecol Res 2019;45:266-79.

7. Morice P, Uzan C, Gouy S, et al. Gynaecological cancers in pregnancy. Lancet 2012;379:558-69.

8. Sardi JE, Giaroli A, Sananes C, et al. Long-term followup of the first randomized trial using neoadjuvant chemotherapy in stage Ib squamous carcinoma of the cervix: the final results. Gynecol Oncol 1997;67:61-9.

9. Chen CA, Cheng WF, Wei LH, et al. Radical hysterectomy alone or combined with neoadjuvant chemotherapy in the treatment of early stage bulky cervical carcinoma. J Formos Med Assoc 2002;101:195-202.

10. Stroup DF, Berlin JA, Morton SC, et al. Meta-analysis of observational studies in epidemiology: a proposal for reporting. Meta-analysis Of Observational Studies in Epidemiology (MOOSE) group. JAMA 2000;283:2008-12.

11. Moher D, Liberati A, Tetzlaff J, et al. Preferred reporting items for systematic reviews and meta-analyses: the PRISMA statement. PLoS Med 2009;6:e1000097.

12. Stang A. Critical evaluation of the Newcastle-Ottawa scale for the assessment of the quality of nonrandomized studies in meta-analyses. Eur J Epidemiol 2010;25:603-5.

13. Wu XL, Tu Q, Faure G, et al. Diagnostic and Prognostic Value of Circulating Tumor Cells in Head and Neck Squamous Cell Carcinoma: a systematic review and meta- analysis. Sci Rep 2016;6:20210.

14. Park JO, Lee SI, Song SY, et al. Measuring response in solid tumors: comparison of RECIST and WHO response criteria. Jpn J Clin Oncol 2003;33:533-7.

15. Li X, Huang K, Zhang Q, et al. Early response to neoadjuvant chemotherapy can help predict long-term survival in patients with cervical cancer. Oncotarget 2016;7:87485-95.

16. Robova H, Rob L, Halaska MJ, et al. High-dose density neoadjuvant chemotherapy in bulky IB cervical cancer. Gynecol Oncol 2013;128:49-53.

17. Liu SP, Yang JX, Cao DY, et al. Efficacy of neoadjuvant cisplatin and 5-flourouracil prior to surgery in FIGO stage IB2/IIA2 cervical cancer. Mol Clin Oncol 2014;2:240-4.

18. Yang L, Guo J, Shen Y, et al. Clinical efficacy and safety of paclitaxel plus carboplatin as neoadjuvant chemotherapy prior to radical hysterectomy and pelvic lymphadenectomy for Stage IB2-IIB cervical cancer. Int J Clin Exp Med 2015;8:13690-8.

19. Park JS, Jeon EK, Chun SH, et al. ERCC1 (excision repair cross-complementation group 1) expression as a predictor for response of neoadjuvant chemotherapy for FIGO stage 2B uterine cervix cancer. Gynecol Oncol 2011;120:275-9.

20. Li R, Lu ST, Si JG, et al. Prognostic value of responsiveness of neoadjuvant chemotherapy before surgery for patients with stage $\operatorname{IB}(2) / \mathrm{IIA}(2)$ cervical cancer. Gynecol Oncol 2013;128:524-9.

21. Shoji T, Takatori E, Saito T, et al. Neoadjuvant chemotherapy using platinum- and taxane-based regimens for bulky stage Ib2 to IIb non-squamous cell carcinoma of the uterine cervix. Cancer Chemother Pharmacol 2013;71:657-62.

22. Xie Q, Liang J, Rao Q, et al. Aldehyde Dehydrogenase 1 Expression Predicts Chemoresistance and Poor Clinical Outcomes in Patients with Locally Advanced Cervical Cancer Treated with Neoadjuvant Chemotherapy Prior to Radical Hysterectomy. Ann Surg Oncol 2016;23:163-70.

23. Wang P, Wang M, Li S, et al. Association of the MDM2 SNP285 Polymorphism with Cancer Susceptibility: A Meta-Analysis. Dis Markers 2016;2016:4585484.

24. Egger M, Davey Smith G, Schneider M, et al. Bias in meta-analysis detected by a simple, graphical test. BMJ 1997;315:629-34.

25. Begg CB, Mazumdar M. Operating characteristics of a rank correlation test for publication bias. Biometrics 1994;50:1088-101.

26. Zhang Y, Xia ZG, Zhu JH, et al. Association of Interleukin-10 $-3575 \mathrm{~T}>\mathrm{A}$ and $-1082 \mathrm{~A}>\mathrm{G}$ polymorphisms 
with non-Hodgkin lymphoma susceptibility: a comprehensive review and meta-analysis. Mol Genet Genomics 2015;290:2063-73.

27. Yang R, Li X, Zhou H, et al. Establishment of a cervical cancer bio-bank for the Chinese population: from projectbased sample collection to routine management. Arch Gynecol Obstet 2015;292:399-404.

28. Buda A, Lissoni AA, Floriani I, et al. Long-Term Clinical Benefits of Neoadjuvant Chemotherapy in Women With Locally Advanced Cervical Cancer: Validity of Pathological Response as Surrogate Endpoint of Survival. Int J Gynecol Cancer 2015;25:1468-75.

29. MacLeod C, O'Donnell A, Tattersall MH, et al. Locally advanced cervix cancer: chemotherapy prior to definitive surgery or radiotherapy. A single institutional experience. Australas Radiol 2001;45:491-5.

30. Cai HB, Chen HZ, Yin HH. Randomized study of preoperative chemotherapy versus primary surgery for stage IB cervical cancer. J Obstet Gynaecol Res 2006;32:315-23.

31. Mori T, Hosokawa K, Sawada M, et al. Neoadjuvant weekly carboplatin and paclitaxel followed by radical hysterectomy for locally advanced cervical cancer: longterm results. Int J Gynecol Cancer 2010;20:611-6.

32. Selvaggi L, Loizzi V, DI Gilio AR, et al. Neoadjuvant chemotherapy in cervical cancer: a 67 patients experience. Int J Gynecol Cancer 2006;16:631-7.

Cite this article as: Hou Q, Shen J, Zhou S, Wei J, Wu M, Chen Q, Yan W, Zhang P, Yue Q, Wang S, Huang K. Early response to neoadjuvant chemotherapy helps decrease recurrence rate of cervical cancer: a systematic review and metaanalysis. Ann Palliat Med 2021;10(6):6092-6103. doi: 10.21037/ apm-20-2004
33. Martinelli F, Bogani G, Ditto A, et al. How often parametrial involvement leads to post-operative adjuvant treatment in locally advanced cervical cancer after neoadjuvant chemotherapy and type $\mathrm{C}$ radical hysterectomy? Eur J Surg Oncol 2015;41:1089-96.

34. Huang K, Li X, Yang R, et al. Prospective cohort study to evaluate the efficacy of taxane plus platinum and CPT11plus platinum regimes and to identify prognostic risk factors in cervical cancer patients. Int J Clin Exp Med 2015;8:15018-29.

35. Hu T, Li S, Chen Y, et al. Matched-case comparison of neoadjuvant chemotherapy in patients with FIGO stage IB1-IIB cervical cancer to establish selection criteria. Eur J Cancer 2012;48:2353-60.

36. Huang K, Sun H, Chen Z, et al. Optimal pathological response indicated better long-term outcome among patients with stage IB2 to IIB cervical cancer submitted to neoadjuvant chemotherapy. Sci Rep 2016;6:28278.

37. Gadducci A, Sartori E, Maggino T, et al. Pathological response on surgical samples is an independent prognostic variable for patients with Stage Ib2-IIb cervical cancer treated with neoadjuvant chemotherapy and radical hysterectomy: an Italian multicenter retrospective study (CTF Study). Gynecol Oncol 2013;131:640-4.

38. Zhu J, Jia W, Wu C, et al. Base Excision Repair Gene Polymorphisms and Wilms Tumor Susceptibility. EBioMedicine 2018;33:88-93. 